\title{
Ionel Buşe
}

\section{Aferim !}

\section{ou l'émasculation de l'altérité}

\begin{abstract}
AFERIM! OR THE EMASCULATION OF ALTERITY
Abstract: Romanian director Radu Jude's movie Aferim! represents a gesture of courage. Our interpretation tries to highlight the idea of the emasculation of otherness, the common disease of all identity struck by the self-sufficiency of its abstract purity. Emasculation is thus the gesture of the absolute symbolic elimination of all creation. Since every creation is, at the same time, Another, by which we build identity, we take our own identity away in that process.
\end{abstract}

Keywords: Romanian Cinema; Radu Jude; Aferim!; Rroma Community; Identity; Diversity; Alterity; Emasculation.

\section{IONEL BUȘE}

Université de Craiova, Roumanie

ionelbuse@yahoo.com

DOI: 10.24193/cechinox.2019.37.24
工 e film roumain Aferim! (2015), réalisé Radu Jude, a été commenté très brièvement en Roumanie malgré l'appréciation du jury du Festival du film de Berlin de 2016 qui lui a décerné l'Ours d'argent du meilleur réalisateur. Parfois, il a fait l'objet d'une critique autochtone influencée par un «politiquement correct " excessif : le film porte sur les Roumains (ce qui signifie sur leurs péchés), mais il n'est pas fait pour les Roumains. Il a même été critiqué pour sa réalisation artistique : "La critique allemande peut féliciter le film Aferim ! en lui donnant un ours ou même deux. Le prix obtenu à Berlin peut même passer pour une des performances notables du film roumain. Mais pour moi, Aferim! le fait est toujours là : un film a été récompensé plus pour sa touche d'ironie et de compassion à l'égard du destin des Tsiganes que pour ses valeurs $\operatorname{artistiques}^{1}$ ». Certaines " approches » dénoncent la mauvaise foi ou l'ignorance $\mathrm{du}$ réalisateur en matière de croyances orthodoxes ou des mentalités : « le film est subordonné d'un bout àl'autre à la soi-disante thèse qui considère la Valachie comme retardataire, sale, concupiscente, chauviniste, blasphématoire, sans idéaux et sans Dieu... Tout cela est imposé aux spectateurs avec 
schématisme, pour démontrer à tout prix une opinion préconçue en produisant des événements et des circonstances pour l'illustrer ${ }^{2} »$. En conséquence, l'habileté du jury à attribuer un prix à un tel film peut être considérée comme discutable :

D'où vient donc le grand Ours d'argent ?... Darren Aronofsky a été le président du jury de cette année, un ignorant en matière de Bible et de mentalités, mais un grand fan des effets spéciaux, qu'on peut trouver superflus dans sa prodigieuse production de Noah. Les autres jurés, venant de tous les coins du monde, moins de l'Europe de l'Est, avaient peu de familiarité avec Byzance, le Phanar et l'orthodoxie. Mais un film qui révèle l'esclavage tsigane mérite a priori un prix, n'est-ce pas ?3

Je n'aurais pas accordé beaucoup d'importance à un tel texte s'il ne provenait pas d'une "spécialiste » (diplômée de l'Académie de théâtre et de cinéma) qui l'a publié dans un journal " orthodoxe ». Bien sûr, il faut respecter son point de vue, mais si on regarde le film seulementpar la grille d'une Valachie chauvine, concupiscente et sans Dieu, il n'est pas exclu qu'il soit marqué $a$ priori par cette obsession. J'avoue que je n'y ai pas vu une lutte entre chrétiens et non chrétiens, ni seulement du chauvinisme ou de la concupiscence. Le détail ne sert pas toujours au diable, comme essaie de nous convaincre ce texte. Au début du XIX siècle l'image de l'Autre était très différente d'aujourd'hui.

Le scénario du film est simple et ressemble à un western américain. Au début du XIX ${ }^{\mathrm{e}}$ siècle, en Valachie, au Sud de la

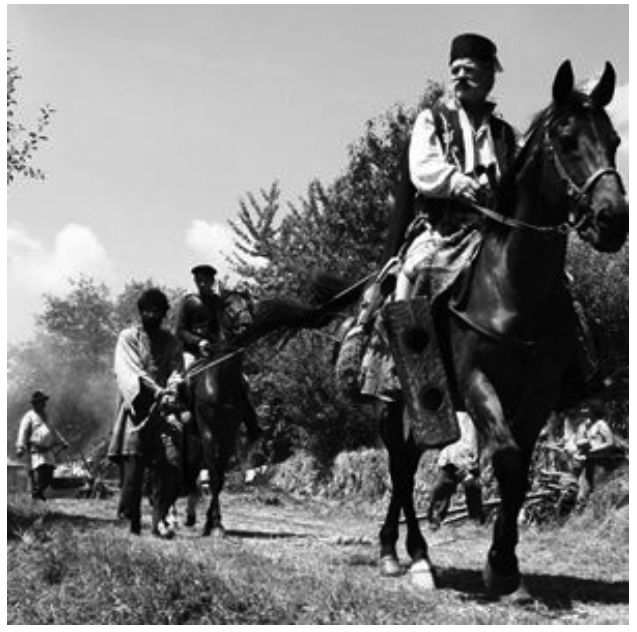

De droite : Constandin (le policier),

Carfin en marche (l'esclave) et Ioniță (le fils de Constandin) - image tirée du film

Roumanie, le policier (zapciul) Constandin, accompagné de son fils, Ioniță, qui est initié à cette occasion à la vie de policier, est embauché par le boyard Iordache pour chercher Carfin, un esclave tsigane, qui a fui après avoir été surpris en train de faire l'amour avec la femme du boyard. Après une longue recherche, Carfin est retrouvé chez un paysan et rendu au boyard qui veut l'émasculer.

La critique élogieuse du film peut être concentrée dans ces remarques :

Dans ce film, au noir et blanc superbe, inspiré par des photographies du $\mathrm{XIX}^{\mathrm{e}}$ siècle, sa vision d'une campagne imprégnée de religiosité, profondément misogyne, ouvertement antisémite et xénophobe, fait écho aux haines toujours vives dans la Roumanie actuelle. Et, particulièrement, à l'intolérance dont sont victimes les Roms, ridiculisés ici par des répliques absurdes et cruelles ${ }^{4}$. 
Le parallèle entre les deux époques est, bien sûr, l'un des buts du film comme l'avoue d'ailleurs le jeune réalisateur. Le film a un caractère militant. Il y a encore aujourd'hui une certaine intolérance dans l'Europe de l'Est concernant les Roms et une certaine misogynie.

Le clou du film est une fête où, dans une ambiance façon Emir Kusturica, l'alcool finit par rapprocher les hommes et les femmes de toutes les origines sociales. Moment savoureux mais éphémère. Car lsultime séquence, drune noirceur terrifiante, rappelle que le racisme conduit forcément à la barbarie ${ }^{5}$.

Aferim! est ainsi l'occasion de remettre en question l'idée d'émasculer la différence. Si comme l'a dit Sartre, dans Huit clos "l'enfer c'est les autres », alors les autres doivent-ils être... castrés ? Dans la dernière séquence du film, l'esclave tsigane Carfin est émasculé par le boyard avec des ciseaux spéciaux réservés aux animaux. Il est castré comme punition pour avoir jeté le doute sur l'autorité masculine du boyard. Peutêtre toute la «philosophie » du film est-elle focalisée ici. L'autre doit être soumis par la castration physique, mais surtout par celle symbolique. Pour être soumis, il faut être vidé de son énergie vitale et de sa liberté. La liberté signifie aussi la possibilité de création et même de procréation.

Au-delà de ces perspectives critiques diverses on peut faire quelques remarques :

1. Le scénario ne prétend pas « reproduire » une époque. Ce serait absurde. C'est une reconstruction artistique, celle qui ressemble au jeu de l'imagination du « comme si », en faisant appel à certains documents, témoignages, images, etc. Les historiens ne le prennent pas trop au sérieux du point de vue de la réalité historique.

2. La création cinématographique est un « point de vue » appartenant à de jeunes cinéastes roumains du siècle $\mathrm{XXI}^{\mathrm{e}}$ siècle (Radu Jude et Florin Lăzărescu) et elle est préparée pour un jury et pour un récepteur sensible à l'idée très actuelle : la question de l'altérité. Le monde doit être vu aussi selon la perspective de la pensée de l'époque. En ce sens, le film peut être considéré comme programmatique, dans le contexte de la radicalisation des grilles du «politiquement correct ». Mais son but n'est pas de déprécier les Roumains, même si leur inculture et leurs mentalités de l'époque sont souvent traitées avec ironie et ridiculisées. La grille est donnée par un modèle de pensée occidentale, c'est vrai, avec ses lumières et ses ombres. Au moins, cette façon de penser librement permet un jugement critique.

3. C'est vrai que les Roumains souffrent de l'image qui leur est conférée par l'Occident, parfois fausse, et qui est exploitée à des fins politiques, mais nous ne pouvons pas limiter une production artistique à un telle visée. Des films plus célèbres révèlent la réalité grossière concernant l'image de l'autre au début du $\mathrm{XIX}^{\mathrm{e}}$ siècle, sur les Indiens américains ou sur la population noire, par exemple. En ce sens, le film Aferim! n'apporte aucune nouveauté. Ou s'il y en a une, elle est pour nous : assumer du point de vue historique la discrimination ethnique, sociale, religieuse, des roumains, etc.

4. Le film est également fait pour nous, roumains. Le national-communisme nous a empoisonnés avec des idéalisations utopiques. Beaucoup d'entre elles sont présentes encore dans des esprits peu instruits. Presque toutes les formes de discrimination ont existé et continuent d'exister. Avant que 
l'étranger nous menace ou nous détruise, nous nous affaiblissons nous-même. À l'ombre du nationalisme peuvent se développer l'identitarisme religieux, le terrorisme et la tyrannie, la corruption et l'insécurité, le manque de la transparence et de la liberté.

Nous ne savons pas si ce type de punition et de violence (l'émasculation) a existé, mais selon divers documents du XIX ${ }^{\mathrm{e}}$ siècle, une telle pratique aurait pu exister. L'esclave fugitif est tsigane et le film se concentre sur l'esclavage des Tsiganes. La plupart des séquences mettent en évidence des images d'esclaves tsiganes ainsi que des Tsiganes libérés qui cherchent des maîtres sur le marché du travail pour pouvoir vivre. Ce n'est pas le "syndrome de Stockholm ", mais une sorte de dépendance dans laquelle le pauvre cherche les moyens de survivre dans les conditions d'une société rurale très pauvre. De ce point de vue, il n'y a pas de grandes différences entre les serfs roumains de Transylvanie, de Valachie, de Moldavie et les esclaves tsiganes. En Transylvanie, par exemple, jusqu'à la fin du XVIII ${ }^{e}$ siècle, les serfs Roumains ont été traité comme des Tsiganes. Ils n'avaient pas le droit de s'approcher de plus de trois cents mètres des domaines des seigneurs. Cependant, il existe des différences entre l'état de servage, dans lequel tombent les paysans qui perdent leur liberté par la servitude, et les Tsiganes qui sont amenés de l'Orient, depuis le début, comme esclaves (achetés et vendus). Dans l'Empire byzantin, la première attestation documentée des communautés tsiganes remonte à 1068 et dans l'espace roumain à 1385 quand plusieurs Tsiganes furent offerts comme esclaves au monastère de Vodița par le prince de Valachie, Vladislav.

Le film se concentre davantage sur ce type d'esclavage car le problème des Roms

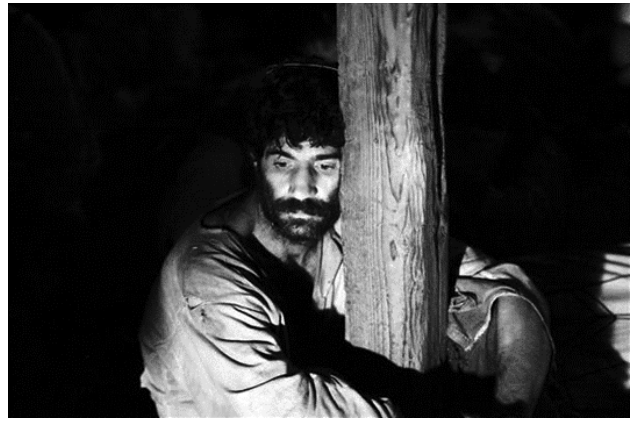

Carfin (l'esclave tsigane pris par le policier) - image du film

est plus connu aujourd'hui en Europe et leur niveau de vie et de formation reste inférieur à celui de la population majoritaire. Il y a des films et des séquences dans lesquels les paysans subissent, d'une manière ou d'une autre, l'abus des autorités, mais le film se concentre sur l'altérité ethnique et pour cette raison a été considéré comme programmatique. Considérant aussi que la deuxième minorité mentionnée dans le film est la minorité juive, nous pouvons mieux comprendre l'intention programmatique du réalisateur. À plusieurs reprises, le Juif est le bouc émissaire, principalement pour son « rôle » dans la condamnation de Jésus. C'est la tradition chrétienne, soutenue par les prêtres, au sujet de la crucifixion, renforcée par quelques aspects de la mythologie populaire pour qui les Juifs sont confondus avec les Géants. Les auteurs du film ont consulté diverses études sur l'image du Juif dans la culture roumaine et, de ce point de vue, le film respecte le canon. L'épisode dans lequel le prêtre explique que le Gitan est plus humain que le Juif parce qu'il descend de Cham alors que le Juif descend des Géants, relève de la même intention.

Si le film était fait de «clichés »? Mais voir partout des clichés n'est-ce pas encore un cliché ? La question de la discrimination 
resterait un lieu commun, un " cliché ", si elle nétait pas si réelle. Qu'on le veuille ou non, nous vivons dans une époque de la revalorisation de l'autre, et ce n'est pas mal. La relation avec l'autre implique de corriger l'intolérance religieuse, la violence domestique ou n'importe quelle forme d'agression. La liberté de pensée et d'expression doit s'imposer d'être responsable et décente. L'histoire se répètera si nous ne reconnaissons pas nos maux. Nous devons apprendre à reconnaître le mal, indépendamment de sa couleur, de sa religion, de sa langue ou de son sexe.

Peut-être le meilleur exemple serait celui de la «femme ». Dans le film, le policier Constandin lit un passage des Écritures à l'épouse du boyard pour prouver que la femme est plus faible et plus bête que l'homme et qu'elle doit lui obéir. Constandin veut convaincre l'épouse du boyard d'admettre sa culpabilité concernant la relation avec Carfin pour épargner à l'esclave fugitif une telle punition cruelle. La femme n'a pas le courage de reconnaître sa culpabilité et le boyard reste insensible aux demandes de Constandin de ne pas émasculer Carfin.

Alors que l'agressivité physique sur les femmes est deux fois plus grande en Roumanie qu'en Europe de l'Ouest, les prêtres orthodoxes continuent d'endoctriner les gens sur l'importance de la soumission " chrétienne » de la femme face à l'homme et des relations sexuelles aux seules fins de la procréation.

En ce qui concerne "l'homophobie ", elle est aussi bien thématisée dans le film. Dans un épisode singulier, celui de l'initiation du jeune Ioniţă à la sexualité, le problème est rapidement expédié par son père qui menace de peine de la mort sa progéniture s'il s'avérait qu'elle ait pratiqué la sodomie, situation que l'on rencontre encore dans l'imaginaire collectif roumain actuel. L'initiation normale à la sexualité doit se faire par une première expérience vénale avec une « prostituée » (tsigane!). L'initiation d'Ioniță dans le monde des adultes est en fait aussi un testament qui préserve un type de mentalité, un monde conservateur dans lequel aucun signe réformiste n'apparaît. Constandin ne fait qu'apprendre à Ioniță comment s'adapter à la vie de l'époque.

Le film Aferim! n'a pas peut-être le prestige et la force artistique d'autres films de la même inspiration, mais dans l'espace roumain il a une importance indiscutable. Pour l'instant nous ne sommes pas dans la situation d'être des modèles pour les autres, quant à la problématique de la différence. Nous sommes encore "pétrifiés" dans un identitarisme post-totalitaire, dans un monde où l'ouverture, l'information et la communication doivent devenir essentielles. De la simple agression du langage dans la rue à l'agression des animaux, des aînés, des enfants, des femmes et même des malades, tout est encore pétrifié. Les craintes qui grandissent systématiquement dans des divers discours religieux radicaux, et dans divers médias, affaiblissent encore notre capacité à répondre lucidement aux différents défis de l'histoire. La manipulation est d'autant plus facile que les gens sont moins informés ou pauvres. La plupart de nos «philanthropes » ne sont que des monstres postcommunistes émotionnellement mutilés. Ils ont plutôt besoin du psychologue que de notre " appréciation ». Aferim! est, tout d'abord, un geste de courage. L'émasculation de l'altérité est la maladie commune de toute logique identitaire frappée par l'autosuffisance de sa pureté abstraite. L'émasculation est le geste 
de l'élimination symbolique absolue de toute création. Et sans création, nous ne rencontrons pas d'altérité qui permettrait de construire une identité. Ce sadomasochisme est à l'origine de notre propre destruction. Voulons-nous multiplier nos visages à l'infini ? Alors, nous choisissons le clonage. À moins que nous ne soyons encore fous, nous deviendrons sûrement fous en nous rencontrant avec nous-mêmes partout.

La plupart des Roumains ont été intrigué par le film documentaire de Channel 4, The Romanians Are Coming. Nous ne sommes pas comme cela, ont répondu les mass-média. Oui, nous sommes aussi comme cela et nous devons reconnaître le bien et le mal en nous, sinon nous ne serons pas en mesure de nous changer, malgré toutes les prières que nous faisons dans nos églises ! Nous ne pouvons pas toujours blâmer l'autre : l'histoire, les Turcs, les Russes, les Hongrois, les Tsiganes, etc. et aujourd'hui, les Anglais, les Français, les Espagnols et les Italiens.

Peut-être l'interprétation la plus juste de ce documentaire de Channel 4 est l'interprétation d'une jeune femme roumaine d'origine Rom qui a fait ses études dans l'enseignement supérieur aux États-Unis ${ }^{6}$.

Je me souviens d'une histoire qui m'a été racontée par mon mentor à Vanderbilt, qui a travaillé de nombreuses années pour la Banque mondiale et qui a visité souvent la Roumanie. Â un moment donné, il était dans un restaurant où on chantait de la musique tsigane de Roumanie. Mon mentor a demandé à une personne qui l'avait accompagné si cette musique faisait partie du répertoire musical roumain. La personne qui avait une position élevée dans le Gouvernement lui a répondu que bien sûr que non, c'est de la musique tzigane, pas de la musique roumaine. Mon mentor lui a répondu : tant que vous ne reconnaissez pas et n'incluez pas les minorités, le pays ne progressera pas. Etre accueillant et inclure d'autres, accepter que les Roms de Roumanie sont roumains, n'est pas un signe de faiblesse, mais de force, d'une la force réelle, par laquelle un changement peut commencer ${ }^{7}$.

Sans aucun doute, un film comme Aferim! pourrait servir à des interprétations simplistes, soutenues par diverses idéologies ou les intérêts politiques ; il est important que nous puissions le juger comme une occasion de repenser l'autre dans le monde d'aujourd'hui où la violence semble de plus en plus présente. La passion dont les réalisateurs font preuve dans leur film est animée par une forme de révolte. Dans le film Django de Tarantino, l'esclave libéré prend sa revanche. Ici, le seul qui prend sa revanche devrait être le film lui-même.

\section{Notes}

1. Cornel Nistorescu, Caricatura românilor din Aferim, Cotidianul, http://www.cotidianul.ro/caricatura-romanilor-din-aferim-258369/, site consulté le 18 mars, 2015.

2. Elena Dulgheru, Aferim și hipsterizarea istoriei, Ziarul lumina, http://ziarullumina.ro/repere-si-idei/ aferim-hipsterizarea-istoriei, site consulté le 4 avril 2015.

3. Ibidem.

4. Samuel Douhaire, Aferim!, Télérama, www.telerama.fr/cinema/films/aferim,498713.php. 


\section{Ibidem.}

6. Cristiana Grigore, boursier Fulbright à l'Université Vanderbilt, chercheur à la Columbia University de New York, a publié des articles in New York Times sur le problème d'assumer l'identité rrome, et a eu des collaborations médiatiques avec Al Jazeera, la radio publique nationale roumaine, Voice of America, etc.

7. Cristina Grigore, Romii din România sunt români, România liberă, http://www.romanialibera.ro/ opinii/comentarii/romii-din-romania-sunt-romani--subiect-inchis-374141, consulté le 7 avril 2015. 